\title{
Incontinencia Urinaria de Esfuerzo Enfoque Actual del Manejo
}

\author{
Dres.: Antonio Lomanto Moran *, \\ Jacinto Sánchez Angarita **
}

El estudio de la Incontinencia Urinaria en la mujer en nuestro medio, es relativamente incipiente, pues las primeras publicaciones se refieren a técnicas vaginales cuyos resultados de aparente eficacia están rodeadas de fallas en el seguimiento, lo que constituye en la actualidad una información de mucha utilidad para valorar resultados.

El hecho de disponer de múltiples técnicas sugeriría que ninguna de ellas es la ideal, pero afortunadamente cada caso requiere de un procedimiento que generalmente se escoge de acuerdo con las experiencias del cirujano.

Aún las técnicas perfectamente concebidas y mejor ejecutadas para la corrección de la Incontinencia Urinaria de Esfuerzo, comprobarán cierta proporción de resultados negativos, y el análisis de estos fracasos pueden dar pautas de enseñanzas para tener en cuenta en procedimientos futuros.

Todavía persiste incertidumbre acerca del procedimiento que otorgue la mejoría definitiva de esa patología con las diferentes técnicas descritas hasta el momento.

El propósito de la presente revisión es el de fijar los criterios que en nuestro Departamento hemos obtenido, fruto de las experiencias con nuestras investigaciones.

Director Departamento de Ginecología y Obstetricia, Facultad de Medicina, Universidad Nacional de Colombia. Jefe de Ginecología Caja de Previsión Distrital, Bogotá D.E.

Profesor Asistente, Departamento de Ginecología y Obstetricia, Facultad de Medicina, Universidad Nacional de Colombia.

\section{VAGINALES}

Son las técnicas que a través de la historia han sido utilizadas con más frecuencia, y con gran disparidad de resultados posiblemente originados por la diversidad del material empleados y tal vez por la dificultad en la aplicación de los últimos procedimientos urodinámicos.

Las técnicas se fundamentan en la suspensión de la uretra, y el cuello, ubicándolo preferiblemente sobre el plano vertical bajo. Los estudios que defienden este procedimiento consideramos un argumento de mucho valor.

El hecho de practicar cirugías simultáneamente (distopias vaginales) durante el acto quirúrgico y su baja estancia hospitalaria pueden favorecer notablemente el procedimiento.

Inicialmente las informaciones obtenidas de la literatura reportaban una alta eficacia, cuestionable ya que en ninguna de las publicaciones se consigna el seguimiento del estudio.

Los argumentos que sustentamos para dudar en la actualidad de las técnicas vaginales en el manejo de la incontinencia urinaria de esfuerzo en la mujer, se basan en el hecho de que la ubicación del cuello vesical no permanece después de realizada la cirugía en el sector intra-abdominal.

La gran mayoría de las mujeres que presentan este tipo de patología son pacientes de alta paridad, cuyos partos en un alto porcentaje no han sido atendidos con las técnicas ideales y es lógico suponer, que las estructuras anatómicas que suspenden el cuello vesical a consecuencia de lo anterior, no tiene la consistencia ideal 
para posteriormente utilizarlas como medio de sostén para la corrección de la incontinencia.

Con base en la poca eficacia que ofrecen estas técnicas, en los últimos decenios se han utilizado aditamentos artificiales como recurso para corregir esa patología.

Es importante anotar que el hecho de comprometer el cuello vesical durante la técnica, no deja de ser un paso preocupante, pues es de todos conocido que los métodos con esta característica ocasionan alta morbilidad.

Además de las observaciones consignadas anteriormente, es bueno tener en cuenta que en aquellos casos en los cuales se ha practicado una preparación vaginal y se intenta practicar algún tipo de intervención, constituye sin discusión alguna dificultades para la disección de esas estructuras, además de correr el riesgo de ocasionar soluciones de continuidad tanto en la uretra como en la vejiga.

Algunos autores principalmente los ginecólogos, han utilizado en la aplicatura del cuello, material absorbible ("a mediano plazo") lo que podría considerarse como una causa de recidiva. Esta observación puede ser fácilmente cuestionada, pues es un criterio, según las normas elementales en técnicas quirúrgicas, que la cicatrización que se logra con cualquier material está fundamentada en la irrigación y en la adherencia de esas zonas cruentas.

Después de realizar minuciosamente revisiones de la literatura, la eficacia de los procedimientos vaginales, nunca sobrepasan la de las otras técnicas empleadas por la corrección de la incontinencia y nos atrevemos a sugerir que la única razón para utilizarla sería en aquellos casos en los cuales no se dispone de los recursos y de los conocimientos necesarios para practicar aquellas $(1,2,3,4,5,6,7,8)$.

\section{SUPRAPUBICOS}

Son aquellos que se practican por vía abdominal, utilizando estructuras anatómicas como fascias y en algunas ocasiones cintas y suturas sintéticas y siempre con el propósito de ascender el cuello vesical, sin utilizar la mucosa vaginal.

En lo que respecta al tipo de incisión somos partidarios de utilizar las que se practican paralelas al arco púbico, -respetando los rectos abdominales- con el propósito de obtener un adecuado campo quirúrgico. No desconocemos que no es la incisión considerada como la más fisiológica puesto que se comprometen las estructuras anatómicas transversalmente, pero también es cierto, que este tipo de intervención es la que ofrece el campo quirúrgico más adecuado. Teniendo en cuenta que el procedimiento se realiza en una zona muy vascularizada, consideramos que el drenaje postoperatorio, por el menor tiempo posible, es de suma importancia para evitar los hematomas ulteriores.

Se han utilizado fascias para levantar el cuello, pero infortunadamente la restricción vascular que ocasiona esta modalidad, necrosa la uretra eventualmente, lo que ha sido una razón más que suficiente para abandonarlas.

La mayoría de ellas, con el fin de identificar la fascia vesicovaginal y el cuello vesical, ocasionan dificultades técnicas, pues el espacio de Retzsius debido a la gran vascularidad, dificulta el'campo quirúrgico,más aun, cuando el cirujano no está familiarizado con el manejo de esta zona.

Los defensores de estos procedimientos suprapúbicos argumentan que utilizando técnicas depuradas identifican fácilmente las estructuras retropúbicas, para así obtener un adecuado ascenso del cuello vesical.

De acuerdo con la bibliografía este tipo de incisiones ofrece el menor número de eventraciones, lo que apoya notablemente el criterio para seleccionarla como la técnica ideal para los procedimientos quirúrgicos abdominales.

Estas técnicas, con las vaginales, han logrado el mayor número de experiencias publicadas en la literatura mundial y han sido objeto de múltiples modificaciones con el propósito de obtener a largo plazo una mejor eficacia y menos efectos colaterales.

Se utiliza la cara posterior del pubis como estructura de anclaje fijando a esta la pared de la uretra, la cara anterior de la vejiga y aún el mismo cuello vesical, que como es de esperarse ocasionan alta morbilidad, no sólo por el hecho de "congelar la uretra" con el riesgo de lesionarla, sino también por la osteitis, que alcanza aproximadamente el 5\%.

Queremos aclarar que según las últimas investigaciones realizadas con el apoyo de la Ingeniería Biomédica utilizando la tecnología más sofisticada se ha podido comprobar que a la uretra, para cumplir su función adecuadamente, se le debe respetar su movilidac por lo menos en sus dos tercios distales lo cual no se cumple con la técnica descrita anteriormente.

Los investigadores interesados en el manejo de la incontinencia urinaria de esfuerzo con el propósito de 
reducir al máximo todas estas complicaciones y para contribuir al éxito de la técnica, resolvieron utilizar la fascia vesicovaginal, cuya disección técnicamente es relativamente fácil y fijarla al ligamento de Cooper. El hecho de utilizar esta estructura, cuya resistencia es altamente favorable, consideramos, ha ofrecido desde el punto de vista estadístico, una eficacia muy superior a las técnicas que emplean el retropubis y con un número menor de efectos indeseables.

De acuerdo con las últimas experiencias realizadas en nuestro medio, el tipo de sutura es fundamental para el éxito de la técnica.

Algunos autores cuestionan la utilización de material no absorbible, argumentando que se corre el riesgo de ocasionar fístulas cuando se compromete parcialmente la uretra y la vejiga.

Esta observación no tiene respaldo alguno, pues si la técnica es adecuada, respetando las estructuras anatómicas, no es posible que se presente este accidente.

En lo relacionado con las complicaciones, no podemos desconocer que el hecho de utilizar drenajes en el espacio de Retzius puede favorecer el riesgo de infección

Otra complicación importante con las técnicas suprapúbicas, es la relacionada con el enterocele, pues al "levantar" la pared vaginal anterior, se corre el riesgo de traccionar el diafragma pélvico ocasionando este tipo de patología. En algunas informaciones aún no publicadas, algunos autores al practicar la corrección de la incontinencia realizan el cierre del fondo de Douglas lo que teóricamente evitaría la aparición del enterocele.

En conclusión; podemos considerar los procedimientos suprapúbicos, como las técnicas con más alta eficacia, a largo plazo, en el manejo de la incontinencia urinaria de esfuerzo en la mujer $(9,10,11,12,13,14$, $15,16,17,18,19)$.

\section{INFRAPUBICOS}

Recientemente descritos, se fundamentan en el acceso vaginal del cuello vesical, para fijarlo con dos puntos, con material no absorbible a la cara posterior del pubis. Sin lugar a dudas al hacer una descripción tan sencilla, consideramos que es la técnica más representativa del grupo y no obstante de existir pocas publicaciones, se constituirían en el procedimiento de elección cuando la mujer presenta cualquier tipo de patología vaginal asociada a incontinencia urinaria de esfuerzo.

La anterior observación la hacemos, pues existen innumerables técnicas para la corrección de la inconti- nencia pero infortunadamente, la mayoría de ellas se caracterizan porque el procedimiento requiere ciertos conocimientos especializados relacionados más con la urología que con la ginecología. Es más, algunos como es el caso de la cervicosuspensión, requieren un paso urológico como es la evaluación endoscópica del cuello y vejiga para comprobar su integridad después de terminar el tratamiento.

Haciendo alusión al comentario anterior, en lo que se refiere a las experiencias con la técnica, en las primeras publicaciones extranjeras el seguimiento de los pocos casos alcanzan los quince años.

En nuestro medio las primeras aparecen en el año de 1983 y en el momento de escribir este artículo, podemos afirmar que la técnica excede el 95\% de éxitos con un seguimiento mayor de cuatro años.

Es una técnica relativamente sencilla para los especialistas que trabajan en el área ginecológica y cuando es practicada con las normas descritas es relativamente más rápida que los procedimientos anteriores.

El hecho de corregir patología preexistente en el sector vaginal como celes de las paredes de la vagina, del útero, uretra y recto, está muy acorde para resolverla durante el acto quirúrgico.

Las complicaciones intraoperatorias son relativamente infrecuentes, pues no obstante que la técnica requiere abrir la fascia endopélvica en donde se encuentra el temido plejo de Santorini, el sangrado no es un accidente frecuente.

Si nos limitamos a la técnica descrita inicialmente, existiría la posibilidad de incurrir en una complicación post-operatoria como es la osteitis por la fijación que se hace en el periostio del retropubis. Con el propósito de evitar esta complicación nos|atrevemos a sugerir no comprometer en la fijación retropúbica las estructuras óseas, sino tomando en lo posible menos periostio. No desconocemos que a largo plazo esta modificación puede ocasionar caida del punto con la consecuente recidiva de la incontinencia.

Teniendo en cuenta que la técnica es completamente vaginal, las complicaciones postoperatorias son similares a las que se hacen por esa vía con una estancia hospitalaria relativamente corta, lo que constituye una característica altamente favorable debido a los altos costos de nuestras instituciones asistenciales.

De acuerdo con las normas establecidas en el estudio de la incontinencia urinaria de esfuerzo en relación al propósito fundamental de las técnicas que es ubicar 
el tercio proximal de la uretra intra-abdominal, podemos afirmar que la uretrocistopexia infrapúbica, aún con las técnicas más depuradas, no alcanza a cumplir con este requisito lo que sería teóricamente un argumento de mucho valor para demeritar la eficacia del procedimiento.

También es conveniente anotar que por el hecho de no utilizar drenajes en las estructuras anatómicas objeto de la reparación, se constituye en factor favorable frente a las técnicas que lo emplean, porque así la incidencia de infecciones es más baja.

En conclusión consideramos la uretrocistopexia infrapúbica, la técnica ideal para corrección de la incontinencia urinaria de esfuerzo en la mujer cuando existen las distopias relacionadas tanto del trayecto uretrovaginal como del útero y de la pared vaginal posterior $\mathrm{y}$ cuando el cirujano domina la técnica adecuadamente. $(20,21,22,23)$.

\section{COMBINADOS}

Son las técnicas que después de identificar el cuello vesical por vía vaginal, logran ascenderlo y suspenderlo con material no absorbible a los rectos abdominales, bajo visión endoscópica.

Inicialmente fue descrita sin la visión endoscópica, lo que ocasionó múltiples complicaciones, siendo la más importante la lesión de uretra y vejiga con las consecuentes fístulas.

Recientemente al adicionar la cistoscopia, se ha logrado erradicar esta complicación y obtener el propósito más importante de las técnicas para corrección de la incontinencia: la ubicación del tercio proximal de la uretra, intraabdominal.

Es un procedimiento relativamente rápido sin descontar el inconveniente de utilizar la endoscopia urológica, lo que constituye una razón para que el ginecólogo que no tiene la experiencia para practicarla necesite el concurso del urólogo.

Como ventaja adicional de la técnica podemos anotar que es como los infrapúbicos, ideal para practicarla cuando hay patología vaginal asociada a la incontinencia urinaria de esfuerzo.

En la descripción inicial de la técnica se usa como sistema de drenaje vesical, la cistostomía suprapúbica, procedimiento invasivo que además de lesionar la vejiga, no es muy aceptado por los urólogos y menos por los ginecólogos en nuestro medio. Sin embargo en nues- tra experiencia, redujo notablemente la infección urinaria post-operatoria y facilitó el control adecuado del residuo vesical lo que a su vez facilitó la estancia, disminuyéndola en tal forma que es la técnica que presenta la menor permanencia hospitalaria en todas las publicaciones revisadas.

Posiblemente por el uso de la endoscopia, el riesgo de lesionar tanto la uretra como la vejiga y lo poco usual de la cistostomía en nuestro medio, no ha sido muy aceptada y aún rechazada por algunos ginecólogos que prefieren las técnicas infrapúbicas.

Es una técnica que tiene pocas complicaciones intraoperatorias. Sin embargo, en el postoperatorio el hecho de ascender excesivamente el cuello vesical ocasiona dolor mucho más intenso que con los otros procedimientos.

Es sin lugar a dudas la técnica ideal a utilizar en aquellos casos de recidivas, antecedentes de cirugías previas, irradiaciones, reintervención, que con otros métodos aumentaría la morbilidad.

Es ideal en aquellos casos en los cuales la obesidad constituye un riesgo cuando se va a practicar la corrección por vía alta.

Un requisito importante para utilizar la cervicosuspensión endoscópica es que los rectos abdominales se caractericen por su buena consistencia, pues constituye prácticamente el soporte más importante para sostener el cuello vesical y así hacerlo intraabdominal.

Es pertinente dejar en claro que la técnica descrita por el autor que le adicionó la endoscopia, utiliza solamente en el sitio de "agarre" del cuello vesical, un centímetro de dacrón para que la sutura que lo levanta no lo "corte" al practicar el ascenso. Teniendo en cuenta que las pacientes que fueron objeto de nuestro trabajo son provenientes de un nivel socio-nutricional relativamente bajo y con el agravante de que sus estructuras anatómicas no son de la consistencia ideal, resolvimos utilizar, también soporte en los rectos. Consideramos que esta modesta modificación puede tener mucho valor en la eficacia a largo plazo.

No obstante los inconvenientes que hemos anotado de la cervicosuspensión endoscópica para practicarla por el ginecólogo, no podemos desconocer que aún con el riesgo que conlleva de lesionar estructuras tan importantes como la uretra y la vejiga nos atrevemos a afirmar que es, la única técnica que bajo visión endoscópica permite ver con exactitud como va a quedar el cuello vesical y además que su ubicación sea totalmente intraabdominal. 
En lo referente a la experiencia con esta técnica sucede algo similar que con las infrapúbicas, pues solamente existen trabajos con un seguimiento que no alcanza los quince años.

En nuestro medio hay publicaciones que alcanzan los cinco años con una eficacia que sobrepasa el $90 \%$ de éxitos.
En conclusión; la cervicosuspensión endoscópica dentro de los procedimientos que actualmente se utilizan para la corrección de la incontinencia urinaria de esfuerzo en la mujer, ocupa un lugar muy importante para aquellos casos en los cuales no se pueden emplear procedimientos por la alta morbilidad que ocasionan, siempre y cuando el especialista no sólo está en capacidad de practicarla sino que disponga de los recursos tecnológicos necesarios para llevar a cabo. $(24,25,26,27$, $28,29,30$ ).
1. ENHORNING. et al. Urethral closure studient with cine roenganography- and simultaneos bladder-urethra pressure recording. Sugery. Gynecology and Obstetrics. 118: 507-616, 1964.

2. KELLY, H.A. Incontinence of urine in women. Urologic and Cutaneos Review 17: 219-293, 1913.

3. PACEY, K. Pathology and repair of genital prolapse. Obs-Gyn 56: 1-15, 1949.

4. ROOS, R.A. et al. Vaginal prolapse and stress urinary incontinence West Virgina Medical Journal of Obst-Gyn 97: 308$315,1967$.

5. LOW, J.A. The management of anatomic urinary incontinence by vaginal repair. American Journal of Obst. Gyn. 97: 308315, 1967.

6. LOMANTO, A.; GOMEZ, P. Operación de Kelly, Rev. Col. Obst, y Gin. Vol. XXXIV, 281983.

7. LOMANTO, A. Incontinencia Urinaria de Esfuerzo en la mujer. Tratamiento. Memorias Primeras Jornadas de Ginecología y Obstetricia. Clínica Fray Bartolomé de las Casas. Bogotá 111, 1984.

8. REDONDO, H.; LOMANTO, A., Incontinencia Urinaria de Esfuerzo: Revisión de la Operación Kelly en 10 años del Instituto Materno Infantil de Bogotá. Trabajo inédito. 1981.

9. BURCH, J.C., Urethrovaginal fixation to Cooper ligament for correction of stress incontinence, cystocele and prolapse. Am. Jou. Obst. \& Gyn. 81: 2811961.

10. BELTRAN, A., Incontinencia Urinaria de Esfuerzo en la mujer. Rev. Col. Obst. y Ginecol. 12: 1081961.

11. BERNAL, E., La Uretrovesicosuspensión de Marshal-Marchetti-Krantz en la corrección de la Incontinencia Urinaria de Esfuerzo. Rev. Col. Obs. y Ginecol. 15: 159, 1964.
12. LOMANTO, A., Evaluación de la Colposistouretropexia en la Incontinencia Urinaria de Esfuerzo. Rev. Col. Obst. y Ginecol. Vol XXXII, 1121982.

13. TANAGHO, E.A., and Cols. La Cistopexia modificada Rev. Col. de Obst. y Gin. 110: 751, 1976.

14. ARENAS BUENAHORA, Cols. La Cistopexia modificada Rev. Col. de Obs. y Gin. Vol. XXXVI 1: 11, 1985.

15. BECERRA, E. Evaluación de la Cistouretropexia Retropúbica Rev. Col. Obs. y Gin. Vol. XXXVI, 6: 380, 1985.

16. TOBON, G. Cols. Tratamiento de la Incontinencia urinaria de esfuerzo por la operación de Burch. Rev. Col. de Obs. y Gin. Vol. XXXII 1: 59, 1981.

17. LOMANTO, A.; SANCHEZ, J. Análisis de fracasos del manejo de la incontinencia urinaria de esfuerzo. Trabajo inédito 1989.

18. LOMANTO, A.; SANCHEZ, J. Uretrocistopexia suprapúbica. Experiencia de 10 años Trabajo de promoción inédito.

19. POWEL, L.C.; J.R. Retropubic urethrocystopexy: Vaginal approach. Am. Jou. Obs. and Gin. 140: 91, 1981.

20. LOPEZ, L.A.; CORZO, J.E. Corrección de la incontinencia urinaria de esfuerzo por medio de la Uretrocistopexia retropúbica por via vaginal Rev. Col. Obst. y Gyn. Vol. XXXIV, 34, 1983.

21. REDONDO, H.; CRUZ, R. Estudio clínico y enfoque terapéutico de la incontinencia urinaria de esfuerzo en la mujer. Rev. Col. Obst. y Gin. Vol. XXXVII. 1986.

22. REDONDO, H.; CRUZ, R. Uretrocistopexia Infrapúbica. Rev. Col. Obst. Gin. Vol. XXXIX. 1988.

23. PEREYRA, A.J.; LEBERTZ, T.B. Combined Uretrovesical Suspensión and Vagino-Urethroplasty for correction of Urinary Stress Incontinence. Obstetrics and Gynecology. 30: 537-546, 1986-87. 
24. STAMEY, T.A.; SCHEFFER, A.J.; CONDOY, C. Clinical and roentgenographic evaluation of endoscopic suspension of the vesical neck for urinary incontinence. Sug Gynecol Obstet. 140: 355-360, 1975.

25. STAMEY, T.A. Endoscopic suspension of vesical neck urinary incontinence. Surg. Gynecol Obst. 136: 547, 554, 1973.

26. STAMEY, T.A. Urinary incontinence in the female Campbell Urology 3: 2272-2293, 1979.

27. STAMEY, T.A. Endoscopic suspension of the vesical neck for urinary incontinence in females, report on 203 consecutive patients. Annals of Surgery. 192 (4) 465-471, 1980.

28. LOMANTO, A.; SANCHEZ, J. La Cervicosuspensión endoscópica en el tratamiento de la incontinencia urinaria de esfuerzo. Informe preliminar Rev. Col de Obst. y Gin. Vol XXXVIII. 5: 331-345 1987.

29. LOMANTO, A.; SANCHEZ J. Experiencia de 100 casos con la cervicosuspensión endoscópica. (1989). Trabajo de Promoción. Universidad Nacional de Colombia. Inédito. 